\title{
The QCD Effective Charge to All Orders
}

\author{
D. Binosi ${ }^{\mathrm{a}}$ and J. Papavassiliou ${ }^{\mathrm{a}}$ \\ ${ }^{a}$ Departamento de Física Teórica and IFIC, Centro Mixto, Universidad de Valencia-CSIC, E-46100, \\ Burjassot, Valencia, Spain
}

Using the pinch technique, we show how to construct the QCD effective charge to all orders.

The extension of the concept of the renormalization-group-invariant and processindependent effective charge from QED to QCD is of special interest for several reasons. First, the existence of a QED-like effective charge, and the ability to identify directly and unambiguously the infinite subset of gluon self-energy-like radiative corrections, is assumed in renormalon analysis of the behavior of the perturbative QCD series at high orders, and in particular, in the study of nonperturbative effects near the QCD mass scale 11. Moreover, such an effective charge could serve as the natural scheme for defining the coupling in the recently proposed "event amplitude generator" based on the light-cone formulation of QCD [2]. Furthermore, when studying the interface between perturbative and non perturbative effects in QCD, one finds it advantageous to use this familiar QED concept, in conjunction with dispersive techniques and analicity properties of the $S$-matrix [3].

In QED, the effective charge construction proceeds as follows 跑: One begins by considering the unrenormalized photon self-energy $\Pi_{\alpha \beta}^{\circ}(q)=$ $q^{2} P_{\alpha \beta}(q) \Pi^{\circ}\left(q^{2}\right)$, where $P_{\alpha \beta}(q)=g_{\alpha \beta}-q_{\alpha} q_{\beta} / q^{2}$ and $\Pi^{\circ}\left(q^{2}\right)$ is a gauge-independent function to all orders in perturbation theory. After Dyson summation, we obtain the (process-independent) dressed photon propagator between conserved external currents $\Delta_{\alpha \beta}^{\mathrm{o}}(q)=\left(g_{\alpha \beta} / q^{2}\right) \Delta^{\mathrm{o}}\left(q^{2}\right)$, with $\Delta^{\circ}\left(q^{2}\right)=-i\left[1+i \Pi^{\circ}\left(q^{2}\right)\right]^{-1}$. The renormalization procedure introduces the standard relations between renormalized and unrenormalized parameters: $e=Z_{e}^{-1} e^{\mathrm{o}}=Z_{f} Z_{A}^{1 / 2} Z_{1}^{-1} e^{\mathrm{o}}$ and $1+i \Pi\left(q^{2}\right)=Z_{A}\left[1+i \Pi^{\circ}\left(q^{2}\right)\right]$, where $Z_{A}\left(Z_{f}\right)$ is the wave-function renormalization constants of the photon (fermion), $Z_{1}$ the vertex renormalization, and $Z_{e}$ is the charge renormalization constant. The Abelian gauge symmetry of the theory gives rise to the fundamental Ward identity (WI) $q^{\alpha} \Gamma_{\alpha}^{\mathrm{o}}(p, p+q)=S_{\mathrm{o}}^{-1}(p+q)-S_{\mathrm{o}}^{-1}(p)$, where $\Gamma_{\alpha}^{\mathrm{o}}$ and $S_{\mathrm{o}}(k)$ are the unrenormalized all orders photon-electron vertex and electron propagator, respectively. The requirement that the renormalized vertex $\Gamma_{\alpha}=Z_{1} \Gamma_{\alpha}^{\circ}$ and the renormalized self-energy $S=Z_{f}^{-1} S_{\mathrm{o}}$ satisfy the same identity, implies $Z_{1}=Z_{f}$, from which immediately follows that $Z_{e}=Z_{A}^{-1 / 2}$. Given these relations between the renormalization constants, and after pulling out the trivial factor $g_{\alpha \beta} / q^{2}$, we can form the renormalization-group-invariant combination, known as the effective charge,

$\alpha\left(q^{2}\right)=\frac{\left(e^{\circ}\right)^{2}}{4 \pi} \Delta^{\circ}\left(q^{2}\right)=\frac{e^{2}}{4 \pi} \Delta\left(q^{2}\right)$.

In $\mathrm{QCD}$, the crucial equality $Z_{1}=Z_{f}$ does not hold, because the WIs are replaced by the more complicated Slavnov-Taylor identities (STIs), involving ghost Green's functions. Furthermore, the gluon vacuum polarization depends on the gauge-fixing parameter, already at the one-loop order. These facts complicate the QCD definition of a universal (process-independent) effective charge [5] (process-dependent effective charges have been considered in [6]). However, the theoretical framework of the pinch technique (PT) [] made this definition possible.

The PT is a well defined algorithm which reorganizes systematically a given physical amplitude into sub-amplitudes, which have the same kinematic properties as conventional $n$-point functions, (propagators, vertices, boxes) but, in addition, are endowed with desirable physical prop- 
erties. Most importantly, they are independent of the gauge-fixing parameter and satisfy naive, (ghost-free) tree-level WIs instead of the usual STIs. The basic observation, which essentially defines the $\mathrm{PT}$, is that there exists a fundamental cancellation, driven by the underlying BecchiRouet-Stora-Tyutin symmetry, which takes place between sets of diagrams with different kinematic properties, such as self-energy, vertex, and box diagrams. This cancellations are activated when longitudinal momenta circulating inside vertex and box diagrams, generate (by "pinching" out internal fermion lines) propagator-like terms; the latter are combined with the conventional selfenergy graphs in order to give rise to an effective gluon self-energy. Recently, the PT has been generalized to all orders in perturbation theory through the collective treatment of entire sets of diagrams [8].

In this talk we will outline the all-order construction of the QCD effective charge. This is best done in the context of the intrinsic PT procedure. The basic idea is that the "pinch graphs", which are essential in canceling the gauge dependences of ordinary diagrams, are always missing one or more (tree-level) propagators corresponding to the external legs of the improper Green's function in question. It then follows that the gauge-dependent parts of such ordinary diagrams must also be missing one or more external propagators. Thus the goal of the intrinsic PT construction is to isolate systematically the parts of the diagrams that are proportional to the inverse propagators of the external legs and simply discard them. In fact, this is absolutely equivalent to the usual ( $S$-matrix) PT construction: discarding these terms is justified because we know that inside an $S$-matrix element they will eventually cancel (to all orders) against similar pieces stemming from the vertices [8]. The important point is that these inverse propagators arise from the STI satisfied by the (all-order) three-gluon vertex appearing inside appropriate sets of diagrams, when it is contracted by longitudinal momenta. Denoting by $\mathbb{\Gamma}_{A_{\alpha} A_{\mu} A_{\nu}}\left(q, k_{1}, k_{2}\right)$, this STI reads [9]

$$
\begin{aligned}
& k_{1}^{\mu} \mathbb{\Gamma}_{A_{\alpha} A_{\mu} A_{\nu}}\left(q, k_{1}, k_{2}\right)= \\
& {\left[i \Delta_{\nu}^{(-1) \rho}\left(k_{2}\right)+k_{2}^{\rho} k_{2 \nu}\right]\left[k_{1}^{2} D\left(k_{1}\right)\right] H_{\rho \alpha}\left(k_{2}, q\right)}
\end{aligned}
$$

$$
-\left[i \Delta_{\alpha}^{(-1) \rho}(q)+q^{\rho} q_{\alpha}\right]\left[k_{1}^{2} D\left(k_{1}\right)\right] H_{\rho \nu}\left(q, k_{2}\right),
$$

where $H$ represents the ghost Green's function appearing in the conventional formalism; at tree level $H_{\alpha \beta}^{[0]}\left(k_{1}, k_{2}\right)=-i g g_{\alpha \beta}$.

Without loss of generality one can choose the Feynman gauge; then, the only source of longitudinal momenta will be the term $\Gamma^{\mathrm{P}}$ appearing in the decomposition $\mathbb{\Gamma}_{A_{\alpha} A_{\mu} A_{\nu}}^{[0]}=\Gamma_{\alpha \mu \nu}^{\mathrm{F}}+\Gamma_{\alpha \mu \nu}^{\mathrm{P}}$ with [7]

$\Gamma_{\alpha \mu \nu}^{\mathrm{F}}=\left(k_{1}-k_{2}\right)_{\alpha} g_{\mu \nu}+2 q_{\nu} g_{\alpha \mu}-2 q_{\mu} g_{\alpha \nu}$,

$\Gamma_{\alpha \mu \nu}^{\mathrm{P}}=k_{2 \nu} g_{\alpha \mu}-k_{1 \mu} g_{\alpha \nu}$.

On the other hand, with the help of the Batalin-Vilkovisky (BV) formalism [10], formulated in the Feynman gauge of the background field method (BFM) [11, one can relate the BFM gluon two-point function $\widetilde{\mathbb{T}}_{\widetilde{A}_{\alpha} \widetilde{A}_{\beta}}(\widetilde{A}$ denotes the background gluon) with the conventional one $\mathbb{\Gamma}_{A_{\alpha} A_{\beta}}$ through the background-quantum identity (BQI)

$$
\begin{aligned}
\widetilde{\mathbb{\Gamma}}_{\tilde{A}_{\alpha} \tilde{A}_{\beta}}(q) & =\mathbb{\Gamma}_{A_{\alpha} A_{\beta}}(q)+2 \mathbb{\Gamma}_{\Omega_{\alpha} A_{\rho}^{*}}(q) \mathbb{\Gamma}_{A^{\rho} A_{\beta}}(q) \\
& +\mathbb{\Gamma}_{\Omega_{\alpha} A_{\rho}^{*}}(q) \mathbb{\Gamma}_{A^{\rho} A^{\sigma}}(q) \mathbb{\Gamma}_{\Omega_{\beta} A_{\sigma}^{*}}(q),(4)
\end{aligned}
$$

where $\mathbb{\Gamma}_{A_{\alpha} A_{\beta}}^{[0]}(q)=-i q^{2} P_{\alpha \beta}(q), \mathbb{\Gamma}_{A_{\alpha} A_{\beta}}^{[n]}(q)=$ $\Pi_{\alpha \beta}^{[n]}\left(q^{2}\right)$, and $\mathbb{\Gamma}_{\Omega A^{*}}$ represents an auxiliary (unphysical) two-point function connecting a background source $\Omega$ with a gluon anti-field $A^{*}$ (see 12] and references therein for details). The crucial observation made in 12 has then been that the STI of Eq.S̊TIbc and the BQI of Eq.B̊QI are related, since the different auxiliary Green's function appearing in them are connected by a Schwinger-Dyson type of relation, which reads

$$
\begin{aligned}
i \mathbb{\Gamma}_{\Omega_{\alpha} A_{\beta}^{*}}(q) & =C_{A} \int H_{\alpha \rho}^{[0]}(q,-k-q) D(k) \times \\
& \times \Delta^{\rho \sigma}(k+q) H_{\beta \sigma}(-q, k+q),
\end{aligned}
$$

where $C_{A}$ denotes the Casimir eigenvalue of the adjoint representation, i.e., $C_{A}=N$ for $S U(N)$, and we have defined the integral measure $\int \equiv \mu^{2 \varepsilon} \int \frac{d^{d} k}{(2 \pi)^{d}}$, with $d=4-2 \varepsilon$ and $\mu$ the 't Hooft mass.

After these observations, we proceed to the construction of the PT gauge independent two- 
point function $\widehat{\mathbb{T}}_{A_{\alpha} A_{\beta}}$; as we will show, it coincides with the corresponding BFM two-point function $\widetilde{\mathbb{N}}_{\widetilde{A}_{\alpha} \widetilde{A}_{\beta} \text {. The one particle irreducible }}$ (1PI) Feynman diagrams contributing to the conventional gluon self-energy in the $R_{\xi}$ gauges can be always separated into three distinct sets: $(i)$ the set of diagrams that have two external (treelevel) three-gluon vertices, and thus can be written schematically (suppressing Lorentz indices) as $\Gamma^{[0]}\left[\mathcal{K}_{2}\right] \Gamma^{[0]}$, where $\mathcal{K}_{2}$ is some kernel; (ii) the set of diagrams with only one external (treelevel) three-gluon vertex, and thus can be written as $\Gamma^{[0]}\left[\mathcal{K}_{1}\right]$ or $\left[\mathcal{K}_{1}\right] \Gamma^{[0]}$; (iii) all remaining diagrams, containing no external three-gluon vertices. Then, if we carry out the decomposition $\Gamma^{[0]} \Gamma^{[0]}=\Gamma^{\mathrm{F}} \Gamma^{\mathrm{F}}+\Gamma^{\mathrm{P}} \Gamma^{[0]}+\Gamma^{[0]} \Gamma^{\mathrm{P}}-\Gamma^{\mathrm{P}} \Gamma^{\mathrm{P}}$ to the pair of external vertices appearing in the diagrams of the set $(i)$, and the decomposition of Eq.(3) to the external vertex appearing in the diagrams of the set (ii), after a judicious rearrangement of the kernels $\mathcal{K}_{2}$ and $\mathcal{K}_{1}$ (together with their statistical factors), relabeling of internal momenta, and taking into account the transversality of the gluon self-energy, the pinching contribution coming from the 1PI diagrams will read

$$
\begin{aligned}
\left\{\mathbb{\Gamma}_{A_{\alpha} A_{\beta}}\right\}^{\mathrm{P}} & =2 i C_{A} \int \frac{1}{k^{2}} \Gamma_{\alpha \mu \nu}^{\mathrm{P}}(q, k,-k-q) \times \\
& \times \Delta_{\sigma}^{\nu}(k+q) \mathbb{\Gamma}_{A_{\beta} A^{\mu} A^{\sigma}}(q, k,-k-q) .
\end{aligned}
$$

Thus, the longitudinal terms $k_{\mu}$ and $(k+q)_{\nu}$ stemming from $\Gamma_{\alpha \mu \nu}^{\mathrm{P}}$ will trigger the STI of Eq.(2). Therefore, the all order generalization of the intrinsic PT will amount to isolating from Eq.1PIPT the terms of the STI of Eq.(2) that are proportional to $\left[\Delta_{\alpha}^{(-1) \rho}(q)\right]$; we will denote such contributions by $\Pi_{\alpha \beta}^{\mathrm{IP}}(q)$. Thus the 1PI diagrams contributing to the gluon self-energy can be cast in the form $\mathbb{\Gamma}_{A_{\alpha} A_{\beta}}(q)=G_{A_{\alpha} A_{\beta}}(q)+\Pi_{\alpha \beta}^{\mathrm{IP}}(q)$.

Notice however that the one particle reducible $(1 \mathrm{PR})$ set $\mathbb{S}$ containing diagrams constructed from strings of lower order self-energy graphs must also be rearranged following the intrinsic $\mathrm{PT}$ procedure, and be converted into the equivalent set $\widehat{\mathbb{S}}$ containing strings involving PT selfenergies. This treatment of the 1PR strings will give rise, in addition to the $\mathrm{PT}$ strings, to $(a)$ a set of contributions which are proportional to the inverse tree-level propagator of the external legs $d^{-1}(q)$ (with $d(q)=-i / q^{2}$ ), and (b) a set of contributions which is effectively 1PI, and therefore also belongs to the definition of the 1PI PT gluon self-energy; we will denote these two sets of contributions collectively by $S_{\alpha \beta}^{\mathrm{IP}}(q)$. Thus the sum of the 1PI and 1PR contributions to the conventional gluon self-energy can be cast in the form

$$
\begin{aligned}
\mathbb{\Gamma}_{A_{\alpha} A_{\beta}}(q)+\mathbb{S}_{\alpha \beta}(q) & =G_{A_{\alpha} A_{\beta}}(q)+\widehat{\mathbb{S}}_{\alpha \beta}(q) \\
& +\Pi_{\alpha \beta}^{\mathrm{IP}}(q)+S_{\alpha \beta}^{\mathrm{IP}}(q) .
\end{aligned}
$$

By definition of the intrinsic PT procedure, we will now discard from the above expression all the terms which are proportional to the inverse propagator of the external legs, thus defining the quantity $R_{\alpha \beta}^{\mathrm{IP}}(q)=\Pi_{\alpha \beta}^{\prime \mathrm{IP}}(q)+S_{\alpha \beta}^{\prime \text { IP }}(q)$, where the primed functions are defined starting from the unprimed ones appearing in Eq.ip2 by discarding the aforementioned terms. Thus, finally, the all orders intrinsic PT gluon self-energy $\widehat{\mathbb{\Gamma}}_{A_{\alpha} A_{\beta}}(q)$, will be defined as

$$
\begin{aligned}
\widehat{\mathbb{\Gamma}}_{A_{\alpha} A_{\beta}}(q) & =G_{A_{\alpha} A_{\beta}}(q)+R_{\alpha \beta}^{\mathrm{IP}}(q) \\
& =\mathbb{\Gamma}_{A_{\alpha} A_{\beta}}(q)-\Pi_{\alpha \beta}^{\mathrm{IP}}(q)+R_{\alpha \beta}^{\mathrm{IP}}(q) .
\end{aligned}
$$

We next proceed to the construction of the quantities $\Pi_{\alpha \beta}^{\mathrm{IP}}(q)$ and $R_{\alpha \beta}^{\mathrm{IP}}(q)$ discussed above, starting from the first one. Using the definition of $\Gamma^{\mathrm{P}}$ of Eq.decomp, together with the STI S̊TIbc (keeping only pinching terms) and the tree-level value of the $H$ Green's function, from Eq.1PIPT we get

$$
\begin{aligned}
\Pi_{\alpha \beta}^{\mathrm{IP}}(q) & =2 i C_{A} \int H_{\alpha \nu}^{[0]}(q,-k-q) D(k) \times \\
& \times \Delta^{\nu \sigma}(k+q) H_{\rho \sigma}(-q, k+q) \mathbb{\Gamma}_{A^{\rho} A_{\beta}}(q),
\end{aligned}
$$

which, using Eq.g̊ppert2, implies $\Pi_{\alpha \beta}^{\mathrm{IP}}(q)=$ $-2 \mathbb{\Gamma}_{\Omega_{\alpha} A_{\rho}^{*}}(q) \mathbb{\Gamma}_{A^{\rho} A_{\beta}}(q)$.

From the $1 \mathrm{PR}$ set of diagrams $\mathbb{S}_{\alpha \beta}$ instead, we need to identify the subset of contributions $S_{\alpha \beta}^{\mathrm{IP}}$ which is effectively 1PI. In what follows we will suppress Lorentz and momentum indices. Now, it can be shown that the only elements of the $1 \mathrm{PR}$ set $\mathbb{S}$ that can contribute to $S^{\mathrm{IP}}$ are the strings that contains at most three self-energy insertions, i.e., the subsets $\mathbb{S}_{2}=\mathbb{\Gamma}_{A A} d \mathbb{\Gamma}_{A A}$ and 
$\mathbb{S}_{3}=\mathbb{\Gamma}_{A A} d \mathbb{\Gamma}_{A A} d \mathbb{\Gamma}_{A A}[13$. The reason for this is that the terms that one needs to add to a string of order $n$ containing more than three self-energy insertions to convert it into a PT string, will be exactly canceled by the conversion into PT strings of other strings of the same order, but containing a different number of insertions. The only time that this will not happen is when the string contains two or three self-energy insertions. In this case we will get

$$
\begin{aligned}
\mathbb{S}_{2}^{[n]}+\mathbb{S}_{3}^{[n]} & \rightarrow \widehat{\mathbb{S}}_{2}^{[n]}+\widehat{\mathbb{S}}_{3}^{[n]}+2 \sum_{m=1}^{n-1} \mathbb{\Gamma}_{\Omega A^{*}}^{[n-m]} \mathbb{\Gamma}_{A A}^{[m]} \\
& +\sum_{m=1}^{n-1} \sum_{\ell=0}^{m-1} \mathbb{\Gamma}_{\Omega A^{*}}^{[n-m]} \mathbb{\Gamma}_{A A}^{[\ell]} \mathbb{\Gamma}_{\Omega A^{*}}^{[m-\ell]} \\
& =\widehat{\mathbb{S}}_{2}^{[n]}+\widehat{\mathbb{S}}_{3}^{[n]}+S^{\prime \operatorname{IP}[n]}
\end{aligned}
$$

On the other hand, we have that $\Pi^{\prime I P}[n]=$ $-2 \sum_{m=1}^{n-1} \mathbb{\Gamma}_{\Omega A^{*}}^{[n-m]} \mathbb{\Gamma}_{A A}^{[m]}, \quad$ so that putting back Lorentz and momentum indices, we get the all order result $R_{\alpha \beta}^{\mathrm{IP}}(q)=$ $\mathbb{\Gamma}_{\Omega_{\alpha} A_{\mu}^{*}}(q) \mathbb{\Gamma}_{A^{\mu} A^{\nu}}(q) \mathbb{\Gamma}_{\Omega_{\beta} A_{\nu}^{*}}(q)$. Thus, making use of the BQI of Eq.B̊QI, we have the identity

$$
\begin{aligned}
\widehat{\mathbb{\Gamma}}_{A_{\alpha} A_{\beta}}(q) & =\mathbb{\Gamma}_{A_{\alpha} A_{\beta}}(q)-\Pi_{\alpha \beta}^{\mathrm{IP}}(q)+R_{\alpha \beta}^{\mathrm{IP}}(q) \\
& =\widetilde{\mathbb{\Gamma}}_{\widetilde{A}_{\alpha} \widetilde{A}_{\beta}}(q) .
\end{aligned}
$$

As a result we can define the unrenormalized PT gluon self-energy as $\mathbb{\Gamma}_{A_{\alpha} A_{\beta}}=$ $q^{2} P_{\alpha \beta}(q) \widehat{\Pi}^{\circ}\left(q^{2}\right)$, where we have shown that $\widehat{\Pi}^{\circ}\left(q^{2}\right)$ is gauge independent to all orders in perturbation theory. Thus, after Dyson summation, we obtain the dressed PT gluon propagator between external conserved currents $\widehat{\Delta}_{\alpha \beta}^{\mathrm{o}}(q)=\left(g_{\alpha \beta} / q^{2}\right) \widehat{\Delta}^{\mathrm{o}}\left(q^{2}\right)$, where $\widehat{\Delta}^{\mathrm{o}}\left(q^{2}\right)=$ $-i\left[1+i \widehat{\Pi}^{\circ}\left(q^{2}\right)\right]^{-1}$. On the other hand, as it has been shown in [8], the bare PT gluon-quark vertex $\widehat{\Gamma}_{\alpha}^{o, a}(q)$ coincides with the corresponding BFM Feynman gauge vertex $\widetilde{\Gamma}_{\alpha}^{\mathrm{o}, a}(q)$ to all orders in perturbation theory, ensuring the validity of the equality $Z_{1}=Z_{f}$ also in the QCD case. Thus, in complete analogy to QED, we can form the renormalization-group-invariant combination

$\alpha_{s}\left(q^{2}\right)=\frac{\left(g^{\circ}\right)^{2}}{4 \pi} \widehat{\Delta}^{\mathrm{o}}\left(q^{2}\right)=\frac{g^{2}}{4 \pi} \widehat{\Delta}\left(q^{2}\right)$ where $g$ is the QCD coupling constant.

In conclusion, we have presented the all-order construction of the effective charge of QCD, by means of the (intrinsic) PT algorithm. This construction allows for the explicit identification of the conformally (in)variant subsets of QCD graphs 14, and lends itself as the natural candidate for studying issues such as dynamical gluon mass generation in a manifestly gauge-invariant way [7].

Acknowledgments: This work has been supported by the CICYT Grants AEN-99/0692 and BFM2001-0262. J.P. thanks the organizers of QCD 02 for their hospitality and for providing a very pleasant and stimulating atmosphere.

\section{REFERENCES}

1. M. Beneke, Phys. Rept. 317 (1999) 1, and references therein.

2. S. J. Brodsky, Acta Phys. Polon. B 32, 4013 (2001); Fortsch. Phys. 50, 503 (2002); hep$\mathrm{ph} / 0111127$.

3. Y. L. Dokshitzer, G. Marchesini and B. R. Webber, Nucl. Phys. B 469 (1996) 93.

4. See, for example, C. Itzykson and J-B. Zuber, Quantum Field Theory (McGraw-Hill, New York, 1980).

5. N. J. Watson, Nucl. Phys. B 494, 388 (1997).

6. G. Grunberg, Phys. Rev. D 46, 2228 (1992).

7. J. M. Cornwall, Phys. Rev. D 26, 1453 (1982); J. M. Cornwall and J. Papavassiliou, Phys. Rev. D 40, 3474 (1989).

8. D. Binosi and J. Papavassiliou, hep$\mathrm{ph} / 0208189$.

9. J. S. Ball and T. W. Chiu, Phys. Rev. D 22 (1980) 2550 [Erratum-ibid. D 23 (1981) 3085].

10. I. A. Batalin and G. A. Vilkovisky, Phys. Lett. B 69 (1977) 309; Phys. Rev. D 28 (1983) 2567

11. L. F. Abbott, Acta Phys. Polon. B 13, 33 (1982); Nucl. Phys. B 185, 189 (1981).

12. D. Binosi and J. Papavassiliou, Phys. Rev. D 66, 025024 (2002); hep-ph/0204308.

13. J. Papavassiliou and A. Pilaftsis, Phys. Rev. Lett. 75, 3060 (1995); Phys. Rev. D 53, 2128 (1996); D. Binosi and J. Papavassiliou, in preparation.

14. S. J. Brodsky, G. P. Lepage and P. B. Macken- 
zie, Phys. Rev. D 28, 228 (1983). 